\title{
Performance Comparison of Invasive Weed Optimization and Particle Swarm Optimization Algorithm for the tuning of Power System Stabilizer in Multi-machine Power System
}

\author{
Ashik Ahmed \\ Assistant Professor \\ EEE Department \\ Islamic University of \\ Technology, Gazipur, \\ Bangladesh
}

\author{
B.M. Ruhul Amin \\ Lecturer \\ EEE Department \\ Bangladesh University of \\ Business and technology, \\ Bangladesh
}

\begin{abstract}
In this paper, two evolutionary algorithms- Invasive Weed Optimization (IWO) based power system stabilizer (PSS) and particle swarm optimization (PSO) based power system stabilizer is designed for multi-machine power system to compare their tuning performances. IWO is a derivative-free real parameter optimization technique that mimics the ecological behavior of colonizing weeds. PSO is also a derivative-free and flexible optimizer which is powered by the behavior of organism, such as bird flocking. Eigen-value based objective function is considered for the tuning of PSSs to enhance system damping of electromechanical mode. The performance of proposed IWO-based PSS and PSO-based PSS is tested and demonstrated under different disturbances for a four machine example power system. The Eigen value analysis and non-linear time domain simulation results shows that both IWO-based PSS and PSO-based design can successfully damp out the oscillations and thus improve the stability of the system. However, the abilities like faster convergence and greater shifting of critical modes to the left of s-plane keeps the choice of IWO based design in front of PSO based design for the system under consideration.
\end{abstract}

\section{General Terms}

Power system stability, Multi-machine system, Optimization.

\section{Keywords}

PSS design, Invasive Weed Optimization, Particle Swarm Optimization, Dynamic stability.

\section{INTRODUCTION}

Newer approaches are being tried by the power system engineers to maximize power transfer among different areas in a stable manner. Usually the transmission networks are overdesigned to run the operation of the overall system safely. Constraints like thermal limit, rotor angle stability limit and voltage stability limit influences the consideration of safety limit [1]. Presence of low frequency oscillation among the interconnected group of generators creates serious threat to the proper operation of the overall system. Usually plant mode and interconnected mode of oscillations with frequency ranges of $0.7-2.0 \mathrm{~Hz}$ and $0.1-0.8 \mathrm{~Hz}$ respectively are observed [2]. Substantial efforts have been made to realize the impact of Power System Stabilizer (PSS) in damping low frequency oscillation and thus improve the small signal stability of power system [3]. The effectiveness of PSSs from both cost and operational point of view has ensured wide use of it by the utilities. Different modern control theory based approaches have been applied to PSS design problem. These contain optimal control, fuzzy \& neuro-fuzzy control, variable structure control and adaptive control [4-7]. It is shown in [8] that appropriate selection of conventional PSS parameters result in satisfactory performance under system disturbances. Sequential and simultaneous tuning based approaches are used in [9]. In sequential design of PSS only one electromechanical mode is considered for damping among many available modes. It is shown that the optimal parameters designed for a certain mode in this fashion can create adverse effect on some other modes of the system and the overall performance may not be optimal. The PSS tuning problem is formulated as non-linear non differentiable optimization problem in [10] which is found to be very hard to solve using traditionally differentiable optimization algorithms. Several random exploration techniques like Genetic Algorithm (GA), Simulated Annealing (SA), Tabu Search (TS), and Evolutionary Programming (EP) has been successfully used [11-15] to optimize the PSS parameters. These techniques have gained acceptance because of their ability and effectiveness of searching an optimal solution in a problem space. A frequency domain based approach [16] is found to be more appropriate where the PSS design problem is formulated as a multi-objective optimization problem and GA is used to optimize them. Even though GA is found to be very satisfactory in searching global or near global optimal result of the problem, the long run-time constraint limits the use of this. Lately a derivative- free, meta-heuristic optimization algorithm named as Invasive Weed Optimization (IWO) is proposed [17] which imitate the ecological behavior of the colonizing weeds. Successfully utilization of IWO has been found since then in different optimization problems like tuning of a robust controller [17], optimal positioning of piezoelectric actuators [18], designing an E-shaped MIMO antenna [20], designing the encoding sequences for DNA computing [21], developing a recommender system [22] and studying electricity market dynamics [23]. PSO was introduced by Kennedy and Eberhart in 1995 [24]. In contrast to the traditional evolutionary algorithms, PSO keeps track of the information regarding both position and velocity of the particle [25]. Several upgraded PSO algorithms are implemented and applied in [26-27].

In this paper the optimizing capability of IWO and PSO is compared for designing the PSSs parameters for obtaining the 
best performance of a multi-machine power system. The problem of PSS design is first formulated as a constrained optimization problem where an eigenvalue based objective function is used for tuning purpose. IWO and PSO algorithms are then utilized to solve the problem. To investigate and compare the performances of these two algorithms an example four machine power system has been considered. Both eigenvalue analysis and nonlinear time domain simulations have been performed to justify their application under different loading conditions.

\section{PROBLEM STATEMENT}

\subsection{System Model and PSS Structure}

A multi-machine power system can be modeled by a system of nonlinear equations of the form

$$
\dot{X}=f(X, U)
$$

where $\mathrm{X}$ is the vector of state variables and $\mathrm{U}$ is the vector of input variables. In this work a two-axis synchronous generator model [28] given in the appendix is used for the simulation purpose. The linearized model of the power system around an operating point is usually employed for PSS design problem. Therefore for an $\mathbf{m}$ machine npss stabilizer power system the state equation of Eq. (1) becomes

$$
\Delta \dot{X}=A \Delta X+B \Delta U
$$

Where, $\mathrm{A}$ is $4 m \mathrm{X} 4 m$ state matrix, $\Delta X$ is $4 m X 1$ state vector, $\mathrm{B}$ is $4 m X$ npss input matrix, $\Delta U$ is npss $X 1$ input vector. Matrix A equals $\frac{\partial f}{\partial x}$, matrix B equals $\frac{\partial f}{\partial u}$; both evaluated at $x_{0}$ which is the operating point around which the system under study is linearized.

In this paper widely used speed based PSS design is considered where the stabilizing signal is assumed to be proportional to the speed. The structure of PSS is shown in Fig. 1. It consists of a gain block with gain $K_{p s s i}$, a signal washout block and two-stage phase compensation blocks. Hence, the transfer function of the $i$ th PSS is given by:

$$
U_{p s s, i}=K_{p s s, i} \frac{s T_{w i}\left(1+s T_{1 i}\right)\left(1+s T_{3 i}\right)}{1+s T_{w i}\left(1+s T_{2 i}\right)\left(1+s T_{4 i}\right)} \Delta \omega_{i}
$$

Where, $\Delta \omega_{i}$ and $U_{p s s i}$ are the deviation of synchronous speed and the stabilizer control signal which is added to the excitation system reference. The signal washout block time constant $T_{w i}$ allows the signal associated with oscillations in rotor speed to pass unchanged, and restricts the steady state changes to modify the terminal voltages. So from the washout block function the value of $T w$ is generally not critical and may be in the range of 0.5 to 20 seconds. In this paper, it is fixed to $10 \mathrm{~s}$. The phase compensation block with time constants $T_{1}, T_{2}$ and $T_{3}, T_{4}$ supplies the required phase-lead to compensate for the phase lag between input and the output signals. The five PSS parameters consisting of the four time constants $T_{1}$ to $T_{4}$ and the gain $K_{p s s}$ need to be optimally chosen for each generator to guarantee optimal system performance under various system configurations and system loadings to ensure the robustness of the technique.

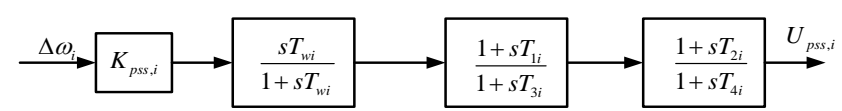

Fig 1: Power system stabilizer structure

\subsection{Objective Function}

To select the best stabilizer parameters that enhance the power system dynamic performance the most, an eigenvalue based objective function is considered as given in Eq. 4.

$$
\mathrm{J}=\text { - min (real (eigenvalues) / abs (eigenvalues ) ) }
$$

So the objective here is to maximize the minimum of the damping ratio for a certain parameter set. Maximizing the minimum damping ratio will help the cause of improving the system overall damping. The problem constraints are the stabilizer parameter bounds in this case. Therefore, the design problem can be formulated as the following optimization problem:

\section{Maximize J}

Subject to,

$$
\begin{aligned}
& K_{i}^{\min } \leq K_{i i} \leq K_{i}^{\max } \\
& T_{1 i}^{\min } \leq \boldsymbol{T}_{1 i} \leq T_{1 i}^{\max } \\
& T_{2 i}^{\min } \leq \boldsymbol{T}_{2 i} \leq T_{2 i}^{\max } \\
& T_{3 i}^{\min } \leq \boldsymbol{T}_{\mathbf{3 i}} \leq \boldsymbol{T}_{\mathbf{3 i}}^{\max } \\
& T_{4 i}^{\min } \leq T_{4 i} \leq T_{4 i}^{\max }
\end{aligned}
$$

where $\mathrm{i}$ is the number of stabilizers considered and $\mathrm{J}$ is the objective function defined in equation (4). In this work both Invasive Weed Optimizer and Particle Swarm Optimizer are used to search for the optimum parameter settings of the given stabilizers and a comparative study is done.

\section{INVASIVE WEED OPTIMIZATION}

Invasive Weed Optimization is a bio-inspired numerical stochastic optimization algorithm that simply simulates natural behavior of weeds in colonizing and finding suitable place for growth and reproduction. Some of the distinctive properties of IWO in comparison with other evolutionary algorithms are the way of reproduction, spatial dispersal, and competitive exclusion [17].

The IWO process begins with initializing a population. That is, a population of initial solutions is randomly generated over the solution space. Then members of the population produce seeds depending on their comparative fitness in the population. In other words, the number of seeds for each member varies linearly between $S_{\min }$ for the worst member and $S_{\max }$ for the best member. These seeds are then randomly scattered over the search space by normally distributed random numbers with mean equal to zero and an adaptive standard deviation. The equation for determining the standard 
deviation (SD) for each generation is presented as: $\sigma_{\text {iter }}=\frac{\left(\text { iter }_{\max }-\text { iter }\right)^{n}}{\left(\text { iter }_{\max }\right)^{n}}\left(\sigma_{\text {initial }}-\sigma_{\text {final }}\right)+\sigma_{\text {final }}$

where iter $_{\max }$ is the maximum number of iterations, $\sigma_{\text {iter }}$ is the $\mathrm{SD}$ at the current iteration and $n$ is the nonlinear modulation index. The produced seeds, accompanied by their parents are considered as the potential solutions for the next generation. Finally, a competitive exclusion is conducted in the algorithm, i.e. after a number of iterations the population reaches its maximum, and an elimination mechanism is adopted. The seeds and their parents are ranked together and those with better fitness survive and become reproductive. A flow chart describing the IWO algorithm is presented in Fig. 2.

\subsection{Selection of IWO Control Parameters}

The convergence of the algorithm depends on the selection of three parameters, the initial $\mathrm{SD}$, ' $\sigma_{\text {initial }}$ ', the final $\mathrm{SD}$, ' $\sigma_{\text {final }}$ ' and the nonlinear modulation index ' $n$ '. These three must be tuned in such a way that the proper value of SD in each iteration is achieved, according to (11). High value of $\sigma_{\text {initial }}$ helps the algorithm search the whole search space aggressively whereas a finer optimum solution can be achieved by decreasing the parameter $\sigma_{\text {final }}$. Maximum and minimum numbers of seeds are the other two criteria than influence the performance of the algorithm. A value between 3 and 5 for the maximum and 0 for the minimum yields good results as found in many examples [28]. Finally the maximum number of plants should also be chosen for IWO. It was found that a value between 10 and 20 gives excellent performance [28].

\section{PARTICLE SWARM OPTIMIZATION}

The particle swarm concept originated as a simulation of simplified social system. The original intent was to graphically simulate the choreography of bird of a bird block or fish school. However, it was found that particle swarm model can be used as an optimizer.

One of the widely used population based optimization approach, Particle Swarm Optimization (PSO) has a good number of advantages over its counterparts. It shares many similarities with the other population based approach like Genetic Algorithm (GA) in initiating the solution by generating some random particles and then updating them in search of the optimum value. Unlike GA, it does not employ any evolutionary operator like mutation and crossover. Rather the particles fly through a predefined search space and with each generation move closer to the optimal value. Compared to GA it has fewer parameters to adjust and easier to implement. One strong feature of PSO is its fast convergence which makes it comparable with other algorithm.

PSO has memory, i.e. all the particles remember its previous best position (local best) as well as the best position of the group (global best). As the initial population is maintained, there is no need for applying operators to the population, which saves both time and memory. Along with that, PSO works on a 'constructive manner' between the particles, in complete contrast with the other artificial algorithms which are based on the concept of "Survival of the Fittest".

The PSO starts with a population of random solution "particles" in an N-dimensional space. The $\mathrm{i}^{\text {th }}$ particle is represented by $\mathrm{X}_{\mathrm{i}}=\left(\mathrm{x}_{\mathrm{i} 1}, \mathrm{x}_{\mathrm{i} 2}, \ldots, \mathrm{x}_{\mathrm{iN}}\right)$. Each particle keeps track of its coordinates in the solution space which are associated with the fittest solution it has achieved so far. The value of the fitness for particle $\mathrm{i}$ (pbset) is also stored as $\mathrm{P}_{\mathrm{i}}=\left(\mathrm{p}_{\mathrm{i} 1}, \mathrm{p}_{\mathrm{i} 2}\right.$, $\left.\ldots, \mathrm{p}_{\mathrm{iN}}\right)$. The global version of the PSO keeps track of the overall best value (gbest), and its corresponding location, obtained thus far by any particle in the population.

PSO consists of, at each step, changing the velocity of each particle toward its pbest an gbest according to equation (12). The velocity of particle i represented as $\mathrm{Vi}=\left(\mathrm{v}_{\mathrm{i} 1}, \mathrm{v}_{\mathrm{i} 2}, \ldots, \mathrm{v}_{\mathrm{iN}}\right)$. Acceleration is weighted by a random term, with separate random numbers being generated for acceleration towards pbest and gbest. The position of the $\mathrm{i}^{\text {th }}$ particle is then updated according to equation (13).

$$
v_{i n}=w^{*} v_{i n}+c_{1}^{*} \operatorname{rand}() *\left(p_{i n}-x_{i n}\right)+c_{2} * \operatorname{rand}() *\left(p_{g n}-x_{g n}\right)
$$

$$
x_{i n}=x_{i n}+v_{i n}
$$

where, $\mathrm{p}_{\mathrm{in}}=$ pbest and $\mathrm{p}_{\mathrm{gn}}=\mathrm{gbest}$

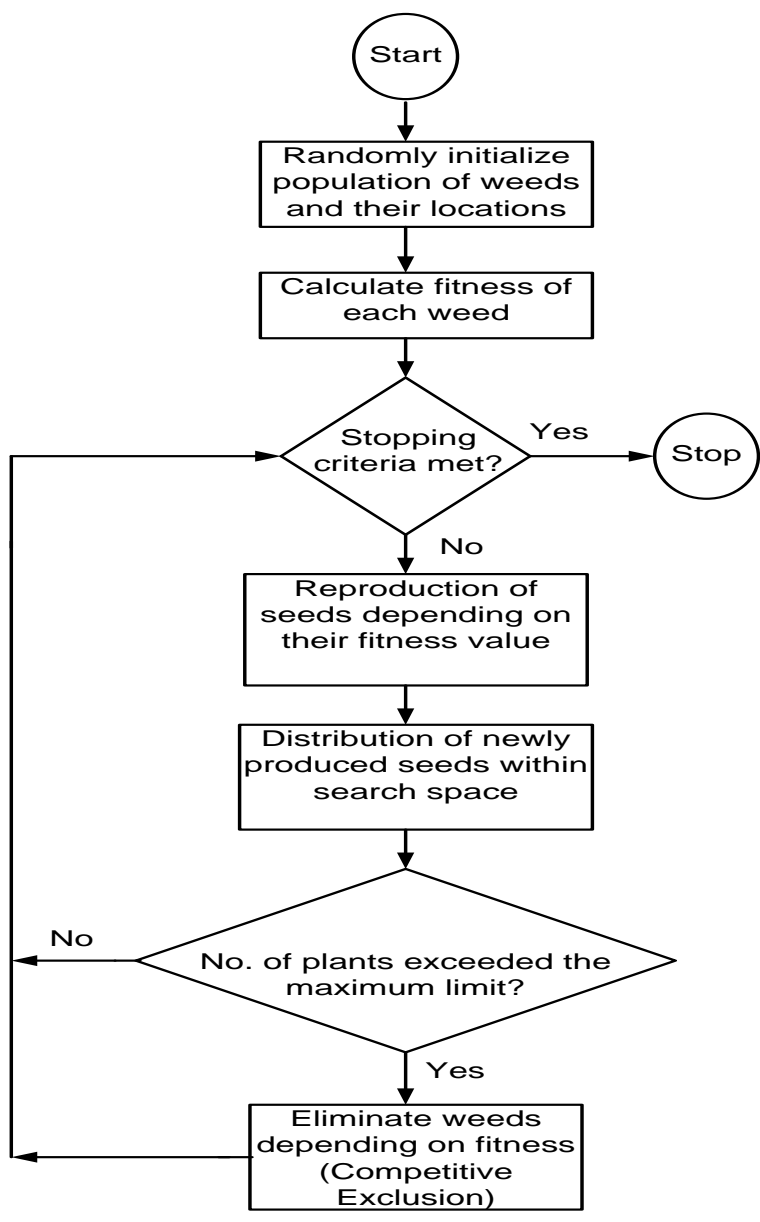

Fig 2: Invasive Weed Optimization algorithm flowchart

\subsection{Steps of PSO algorithm}

Step 1: Define the problem space and set the boundaries, i.e. equality and inequality constraints. 
Step 2: Initialize an array of particles with the random positions and their associated velocities inside the problem space.

Step 3: Check if the current position is inside the problem space or not. If not, adjust the positions so as to be inside the problem space.

Step 4: Evaluate the fitness of each particle.

Step 5: Compare the current fitness value with the particle's previous best value (pbest []). If the current fitness value is better, assign the current value to pbest [] and assign the current coordinates to pbest $x[][n]$ coordinates.

Step 6: Determine the current global minimum among the particle's best position.

Step 7: If the current global minimum is better than gbest, assign the current value to gbest[] and assign the current coordinates to gbest []$[\mathrm{n}]$ coordinates.

Step 8: Change the velocities according to equation (12).

Step 9: Move each particle to the new position according to equation (13) and return to Step 3.

Step 10: Repeat Step3-Step9 until the stopping criteria is satisfied.

\subsection{Selection of PSO Control Parameters}

The convergence of the PSO algorithm depends on the selection of the initial inertia weight and maximum allowable velocity. In this work the initial inertial weight and number of intervals in each space dimensions are being taken as 0.9 and 30 respectively. The number of particles and decrement constant are set at 20 and 0.99 correspondingly. The algorithm was set to stop if, i) the number of iterations reaches 500 or, ii) there is no change in gbest for the last 50 iterations.

\section{SIMULATION RESULTS}

The single-line diagram of two-area, 4-machine test system, as shown in Figure 3, is used to examine both local and inter area oscillations control problems. The system consists of four generators, G1 and G2 for area 1 and G3 and G4 for area 2. The system loads L7 and L9 are present at buses 7 and 9 respectively. Detail system data can be obtained from [29]. For illustration and comparison purpose all four generators are assumed to have PSSs installed. It is also decided that all four time constants for each PSS along with the PSS gain will be tuned to obtain the optimum value. So the number of parameters to be optimized for this work is 20 . The maximum and minimum ranges for the gains and different time constants for optimization purpose are set at [0-50] and [0.011.0] respectively. The IWO and PSO algorithms are then applied to find out the optimized value of parameters for the objective function in (4). The final values of the optimized parameters for IWO and PSO are listed in Table II and III respectively.

\subsection{Eigen value Analysis and Nonlinear Simulation Results}

Three different cases as nominal, light and heavy loading are considered over a wide range of loading conditions for the comparison purpose. The system loading levels at these cases are given in Table I. The IWO algorithm runs several times and then optimal set of PSS parameters are selected. The eigenvalues and the damping ratio of electromechanical modes without PSS is given in Table IV, the eigenvalues and the damping ratio of electromechanical modes with IWObased PSS and PSO-based PSS are shown in Table V and VI.

From table IV we observe that without the PSS installed there are few eigenvalues in the right side of s-plane which will cause severe oscillation in the system. This fact is clear from the fact that an eigenvalue in the right half plane exhibits negative damping ratio and thus deteriorates the damping performance of the overall system. The positive eigenvalues along with their negative damping ratios are highlighted in Table IV. From Table V and VI it is found that both IWO and PSO based PSS can successfully remove the negative damping impact by shifting all the eigenvalues to the left of splane. Furthermore, for the nominal loading case the maximum damping ratio for IWO-PSS is 4.24 and that for the PSO-PSS is 0.9858 . The superiority of IWO based PSS is also confirmed for non-nominal loading conditions, too. The convergence of IWO and PSO algorithms are shown in figures 4 and 5 respectively. It is found that PSO converges more rapidly compared to IWO for all three loading levels considered in this paper.

For illustration purpose a three-phase bolted fault at bus 7 near the end of line 5-7 is considered for the non linear time domain simulations. The different loading conditions considered here are the same for the eigenvalue analysis. The rotor angle variations, angular speed variations and active power variations for nominal, heavy and light loading for Gen 2 are shown in Fig. 6-14. The stabilizer control signal for different case scenarios are shown in Fig. 15-17. From these figures it can be concluded that both IWO and PSO are robust enough to overcome the severe fault conditions and restore the system states to their original levels within a very short duration under different loading levels.

Table I: Loading conditions (in per unit)

\begin{tabular}{|c|c|c|c|c|c|c|}
\hline \multirow{2}{*}{ Load } & \multicolumn{2}{|c|}{ Nominal } & \multicolumn{2}{c|}{ Heavy } & \multicolumn{2}{c|}{ Light } \\
\cline { 2 - 7 } & $\mathrm{P}$ & $\mathrm{Q}$ & $\mathrm{P}$ & $\mathrm{Q}$ & $\mathrm{P}$ & $\mathrm{Q}$ \\
\hline $\mathrm{A}$ & 17.67 & -2.50 & 20.00 & -3.50 & 15.67 & -2.50 \\
\hline $\mathrm{B}$ & 9.67 & -1.00 & 9.67 & -1.00 & 9.67 & -1.00 \\
\hline
\end{tabular}

Table II: Optimal IWO-based PSS parameters with objective function

\begin{tabular}{|c|c|c|c|c|c|c|}
\hline \multirow{2}{*}{ er } & & & & & & Load \\
\hline & $\mathrm{K}$ & $\mathrm{T}_{1}$ & $\mathrm{~T}_{2}$ & $\mathrm{~T}_{3}$ & $\mathrm{~T}_{4}$ & \\
\hline $\mathrm{G}_{1}$ & 45.6522 & 0.0832 & 0.3830 & 0.8059 & 0.0100 & \multirow{4}{*}{ Nominal } \\
\hline $\mathrm{G}_{2}$ & 11.5510 & 0.2823 & 0.3637 & 0.7101 & 0.5891 & \\
\hline $\mathrm{G}_{3}$ & 40.7216 & 0.1494 & 0.0580 & 0.3501 & 0.4679 & \\
\hline $\mathrm{G}_{4}$ & 32.6851 & 0.2146 & 0.6437 & 0.4617 & 0.0100 & \\
\hline $\mathrm{G}_{1}$ & 43.7651 & 0.0933 & 0.4003 & 0.7565 & 0.0100 & \multirow{4}{*}{ Light } \\
\hline $\mathrm{G}_{2}$ & 21.7023 & 0.4274 & 0.6020 & 0.6774 & 0.5842 & \\
\hline $\mathrm{G}_{3}$ & 31.0260 & 0.3345 & 0.8982 & 0.9922 & 0.6685 & \\
\hline $\mathrm{G}_{4}$ & 32.0563 & 0.1162 & 0.7226 & 0.9368 & 0.0100 & \\
\hline $\mathrm{G}_{1}$ & 43.2862 & 0.3769 & 0.6014 & 0.1388 & 0.0606 & \multirow{4}{*}{ Heavy } \\
\hline $\mathrm{G}_{2}$ & 23.9369 & 0.6120 & 0.3851 & 0.1756 & 0.0100 & \\
\hline $\mathrm{G}_{3}$ & 50.0 & 0.1461 & 0.3731 & 0.5570 & 0.0100 & \\
\hline $\mathrm{G}_{4}$ & 19.0656 & 0.3590 & 0.3761 & 0.5329 & 0.5408 & \\
\hline
\end{tabular}


Table III: Optimal PSO-based PSS parameters with objective function

\begin{tabular}{|c|c|c|c|c|c|c|}
\hline \multirow{2}{*}{ Gen } & & & & & & Load \\
\hline & $\bar{K}$ & $\mathrm{~T}_{1}$ & $\mathrm{~T}_{2}$ & $T_{3}$ & $\mathrm{~T}_{4}$ & \\
\hline $\mathrm{G}_{1}$ & 25.1278 & 0.7046 & 0.5180 & 0.2525 & 0.2510 & \multirow{4}{*}{ Nominal } \\
\hline $\mathrm{G}_{2}$ & 32.2625 & 0.7938 & 0.0100 & 0.2381 & 0.7352 & \\
\hline $\mathrm{G}_{3}$ & 45.933 & 0.2244 & 0.7593 & 0.6734 & 0.0558 & \\
\hline $\mathrm{G}_{4}$ & 34.9249 & 0.3479 & 0.7863 & 0.7213 & 0.6287 & \\
\hline $\mathrm{G}_{1}$ & 25.7064 & 0.3754 & 0.1750 & 0.4922 & 0.2970 & \multirow{4}{*}{ Light } \\
\hline $\mathrm{G}_{2}$ & 12.6109 & 0.3848 & 0.7143 & 0.5770 & 0.7505 & \\
\hline $\mathrm{G}_{3}$ & 18.4842 & 0.5608 & 0.1995 & 0.8107 & \begin{tabular}{|l|}
0.6669 \\
\end{tabular} & \\
\hline $\mathrm{G}_{4}$ & 5.7285 & 0.7553 & 0.5024 & 0.4345 & 0.5782 & \\
\hline$\overline{\mathrm{G}_{1}}$ & 19.1365 & 0.1217 & 0.2903 & 0.6341 & 0.0599 & \multirow{4}{*}{ Heavy } \\
\hline $\mathrm{G}_{2}$ & 12.7617 & 0.7039 & 0.6842 & 0.3510 & 0.5055 & \\
\hline $\mathrm{G}_{3}$ & 20.9238 & 0.3146 & 0.3774 & 0.4985 & 0.5637 & \\
\hline$\overline{\mathrm{G}_{4}}$ & 29.5106 & 0.7487 & 0.0180 & 0.0842 & 0.5464 & \\
\hline
\end{tabular}

Table IV: Eigen values and damping ratios without PSS

\begin{tabular}{|c|c|c|}
\hline Nominal & Heavy & Light \\
\hline$-0.589 \pm 7.463 i, 0.078$ & $-89.88 \pm 0.061 i, 1471.2$ & $\mathbf{0 . 0 9 1} \pm \mathbf{4 . 0 4 7 i},-\mathbf{- 0 . 0 2 2}$ \\
$-0.515 \pm 7.292 \mathrm{i}, 0.070$ & $\mathbf{0 . 1 2 7} \pm \mathbf{4 . 0 6 8 i}, \mathbf{- 0 . 0 3 1}$ & $-0.706 \pm 7.303 \mathrm{i}, 0.096$ \\
$\mathbf{0 . 1 0 4} \pm \mathbf{4 . 0 5 1 i},-\mathbf{- 0 . 0 2 5}$ & $-0.343 \pm 7.656 \mathrm{i}, 0.044$ & $-0.535 \pm 7.278 \mathrm{i}, 0.074$ \\
& $-0.514 \pm 7.303 \mathrm{i}, 0.070$ & \\
\hline
\end{tabular}

Table V: Eigen values and damping ratios with IWO-based PSS

\begin{tabular}{|c|c|c|}
\hline Nominal & Heavy & Light \\
\hline$-31.59 \pm 40.93 \mathrm{i}, 0.772$ & $-41.88 \pm 20.94 i, 2.00$ & $-29.01 \pm 41.70 \mathrm{i}, 0.696$ \\
\hline$-7.22 \pm 14.88 \mathrm{i}, 0.485$ & $-29.15 \pm 7.52 \mathrm{i}, 3.876$ & $-5.95 \pm 13.43 \mathrm{i}, 0.443$ \\
\hline$-5.83 \pm 10.26 \mathrm{i}, 0.568$ & $-4.99 \pm 11.33 \mathrm{i} \quad 0.440$ & $-7.34 \pm 10.32 \mathrm{i}, 0.711$ \\
\hline$-2.48 \pm 4.10 \mathrm{i}, \quad 0.605$ & $-2.08 \pm 5.28 \mathrm{i}, \quad 0.394$ & $-7.03 \pm 4.31 \mathrm{i}, \quad 1.631$ \\
\hline$-4.41 \pm 2.90 \mathrm{i}$ & $-1.62 \pm 3.69 \mathrm{i}$ & $-1.78 \pm 2.58 \mathrm{i}$ \\
\hline$-1.55 \pm 1.99 \mathrm{i}$ & $-1.33 \pm 1.15 \mathrm{i}$ & $-2.04 \pm 0.23 \mathrm{i}$ \\
\hline$-1.06 \pm 0.25 \mathrm{i}$ & & $-1.37 \pm 0.27 \mathrm{i}$ \\
\hline & & $-1.10 \pm 0.09 \mathrm{i}$ \\
\hline
\end{tabular}

Table VI: Eigen values and damping ratios with PSO-based PSS

\begin{tabular}{|c|c|c|}
\hline Nominal & Heavy & Light \\
\hline$-4.05 \pm 13.15 \mathrm{i}, 0.2945$ & $-29.30 \pm 18.06 \mathrm{i}, 0.8512$ & $-5.46 \pm 13.74 \mathrm{i}, 0.369$ \\
$-2.74 \pm 11.79 \mathrm{i}, 0.2263$ & $-10.58 \pm 14.24 \mathrm{i}, 0.5967$ & $-3.16 \pm 11.32 \mathrm{i}, 0.268$ \\
$-5.47 \pm 3.422 \mathrm{i}, 0.8479$ & $-6.518 \pm 8.766 \mathrm{i}, 0.5967$ & $-2.35 \pm 3.808 \mathrm{i}, 0.526$ \\
$-2.326 \pm 3.43 \mathrm{i}, 0.5606$ & $-7.778 \pm 2.524 \mathrm{i}, 0.9512$ & $-1.74 \pm 3.215 \mathrm{i}, 0.477$ \\
$-3.467 \pm 0.45 \mathrm{i}, 0.9917$ & $-6.062 \pm 3.529 \mathrm{i}, 0.8642$ & $-2.63 \pm 1.088 \mathrm{i}, 0.924$ \\
$-2.240 \pm 1.37 \mathrm{i}, 0.8522$ & $-2.472 \pm 3.326 \mathrm{i}, 0.5967$ & $-1.25 \pm 0.375 \mathrm{i}, 0.957$ \\
$-1.446 \pm 0.24 \mathrm{i}, 0.9858$ & $-2.331 \pm 3.133 \mathrm{i}, 0.5968$ & \\
& $-1.570 \pm 0.286 \mathrm{i}, 0.9838$ & \\
\hline
\end{tabular}

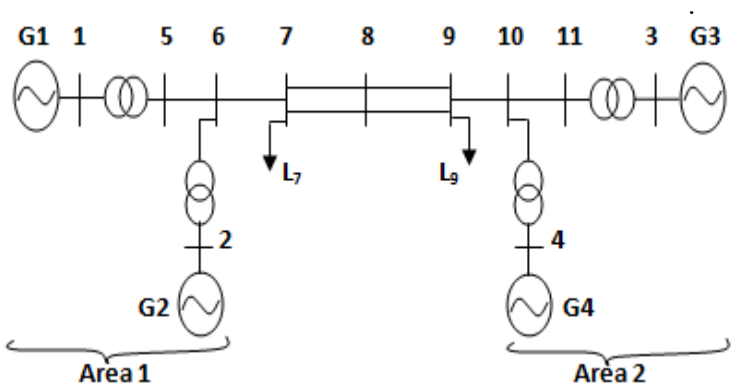

Fig 3: Four-Machine, Two Area System

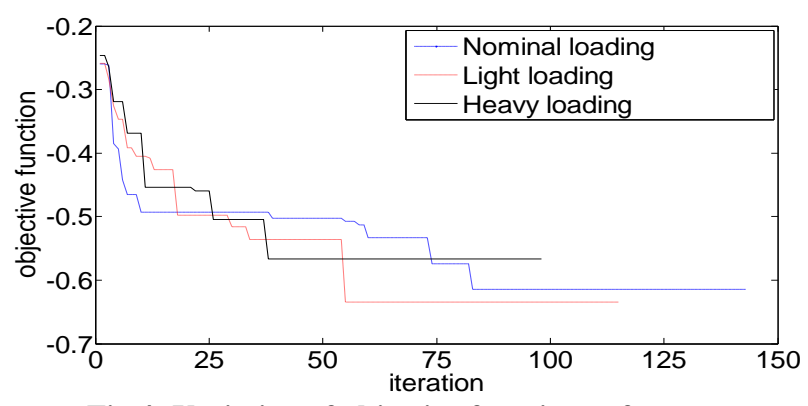

Fig 4: Variation of objective function, $J$ for IWO-based PSS

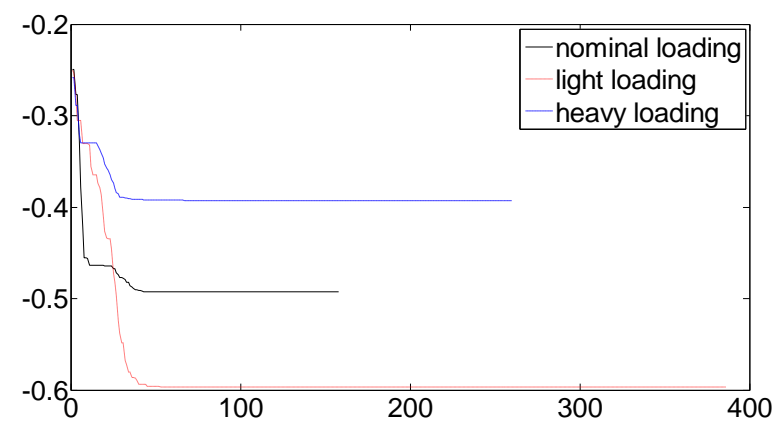

Fig 5: Variation of objective functions, $J$ for PSO-based PSS

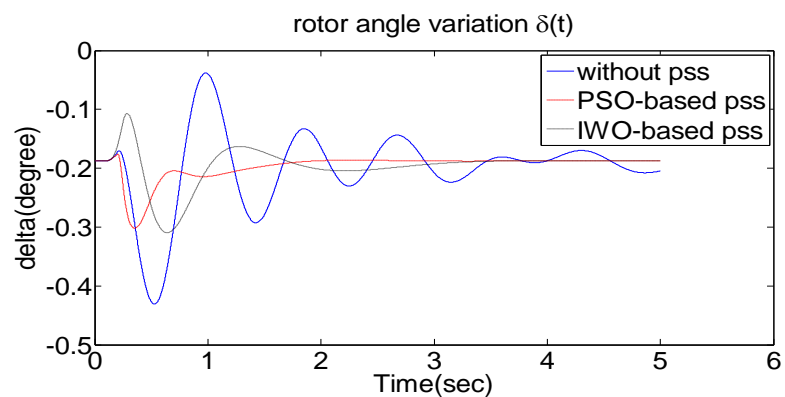

Fig 6: Rotor angle response in nominal

loading for Gen2 


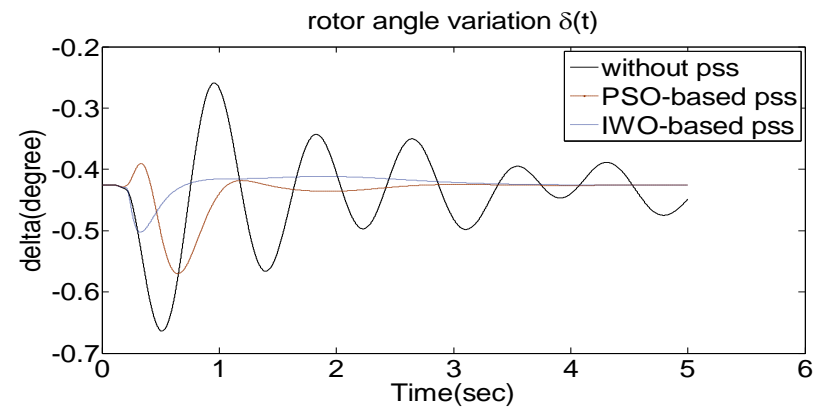

Fig 7: Rotor angle response in heavy loading for Gen2

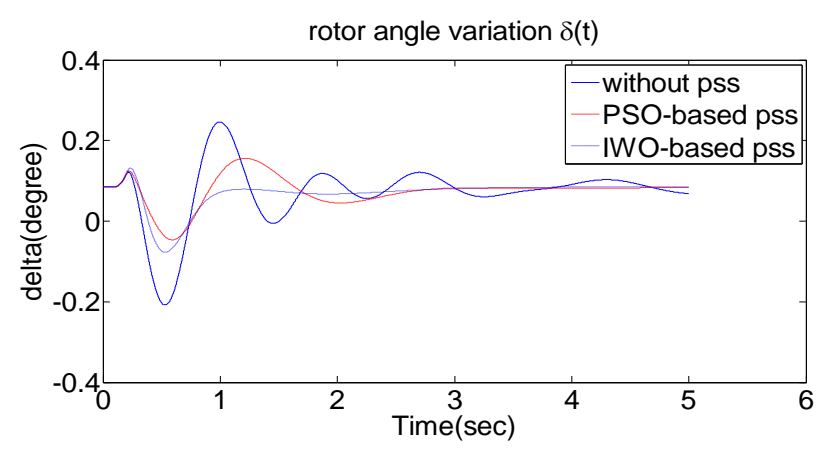

Fig 8: Rotor angle response in light loading for Gen2

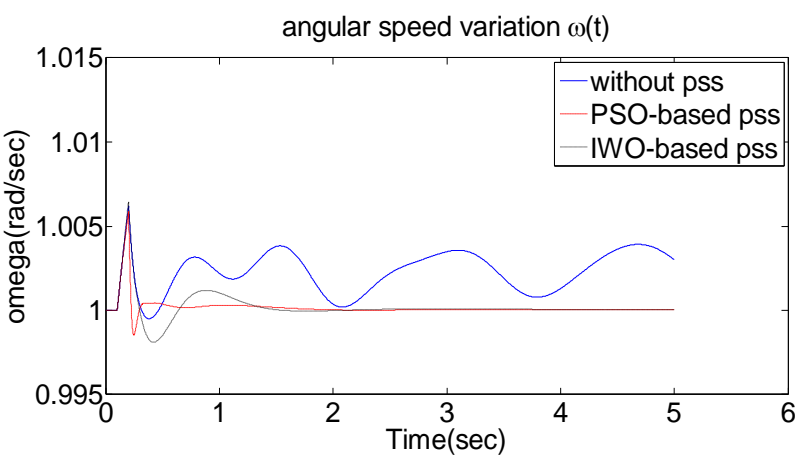

Fig 9: Rotor speed response in nominal loading for Gen2

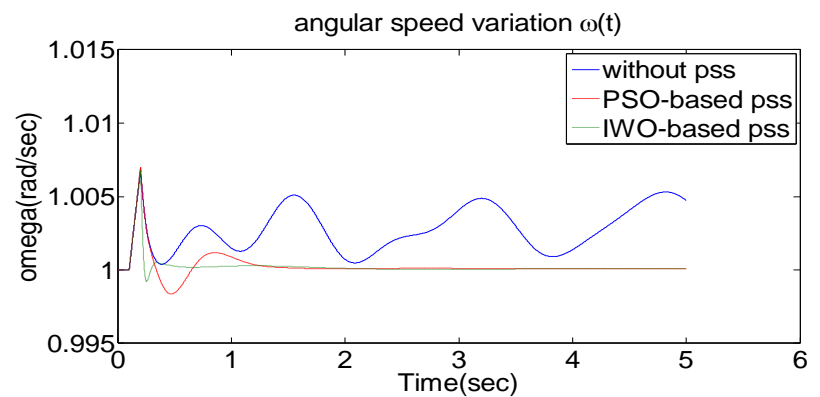

Fig 10: Rotor speed response in heavy loading for Gen2

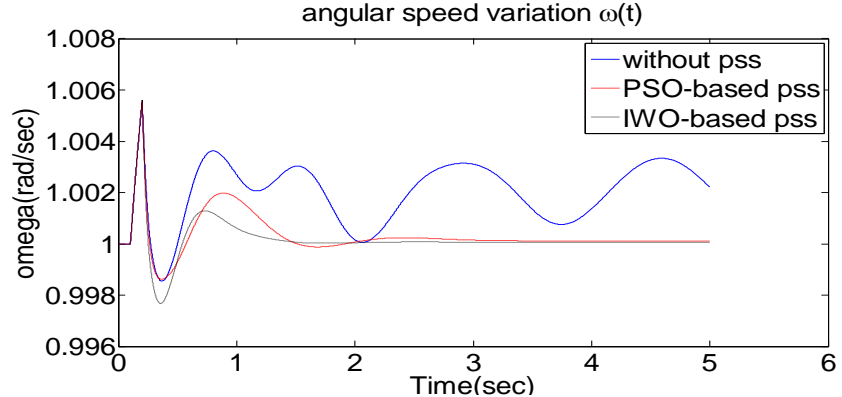

Fig 11: Rotor speed response in light loading for Gen2

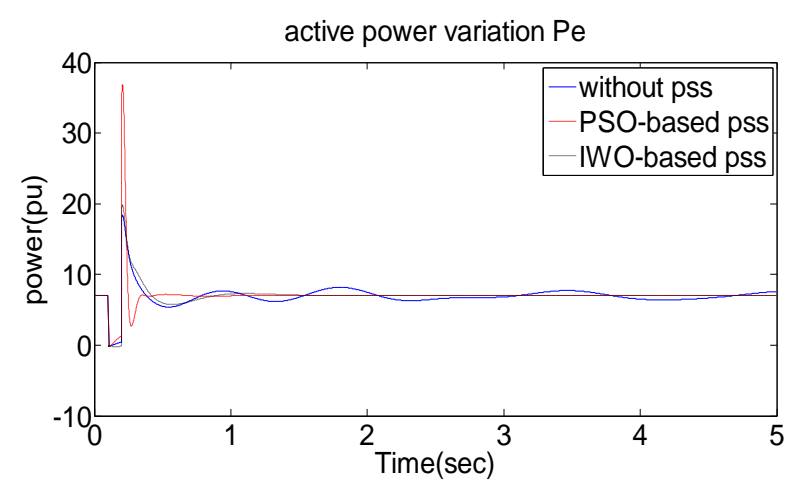

Fig 12: Active power output in nominal loading for Gen2

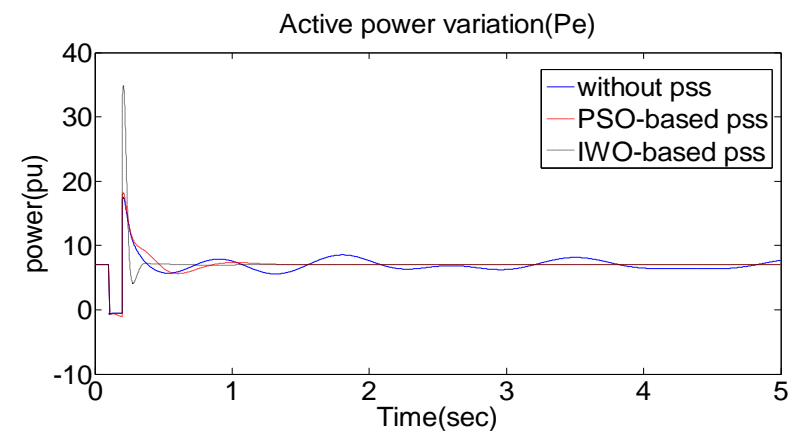

Fig 13: Active power output in heavy loading for Gen2

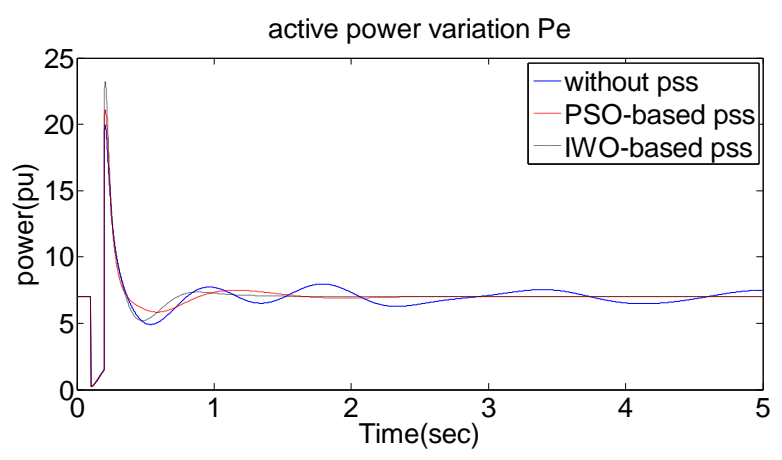

Fig 14: Active power output in light loading for Gen2 


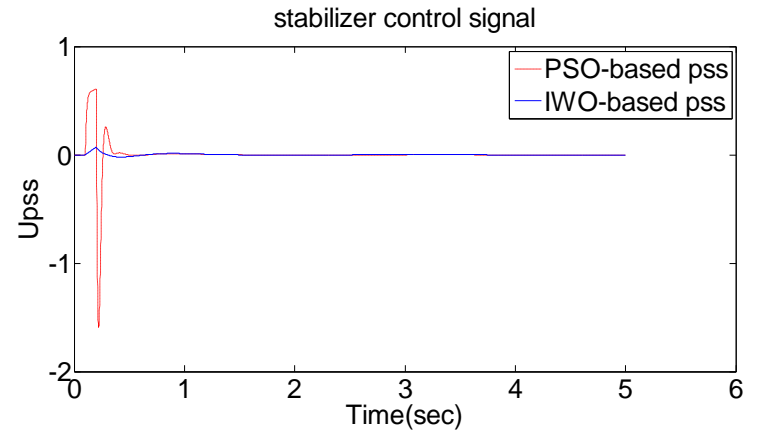

Fig 15: Stabilizer control signal (Upss) in nominal loading for Gen 2

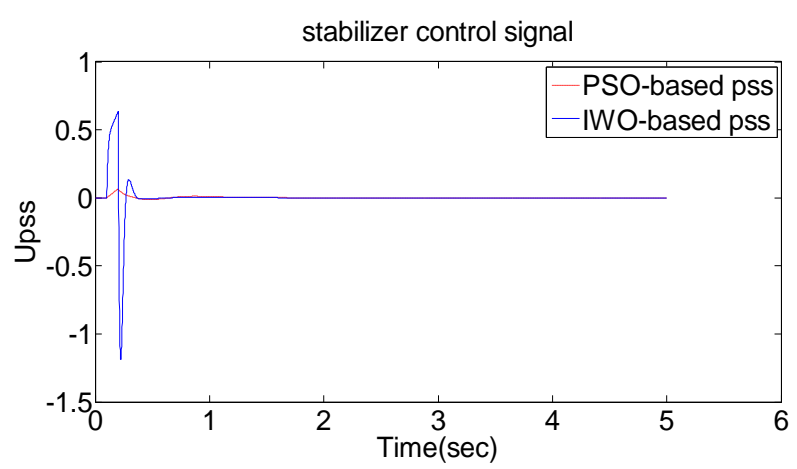

Fig 16: Stabilizer control signal (Upss) in heavy loading for Gen 2

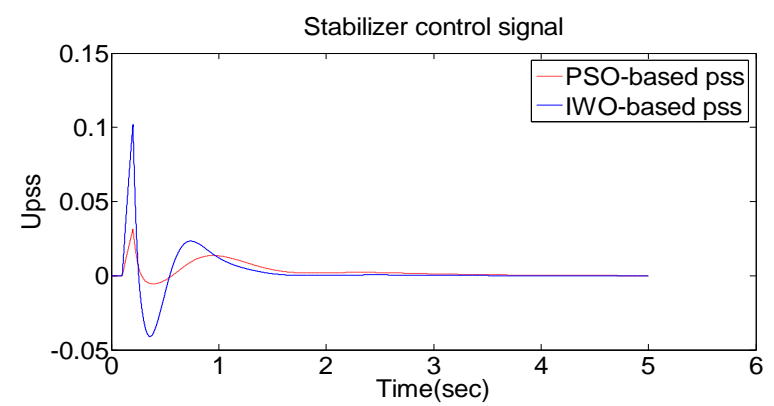

Fig 17: Stabilizer control signal (Upss) in heavy loading for Gen 2

\section{CONCLUSION}

In this paper the performance comparison of the Invasive Weed Optimization and Particle Swarm Optimization technique is investigated for the design of Power System Stabilizers for the damping of low frequency oscillation of a four machine example power system. An eigenvalue value objective function is selected for optimizing the PSS performance. Both eigenvalue based and non-linear time domain simulation is carried out for three different loading conditions. It is found that IWO algorithm converges quicker than the PSO. Eigenvalue based analysis shows that the shifting of the critical modes to the left of s-plane is more in case of IWO compared to PSO. The nonlinear time domain simulation conforms to the results obtained in the eigenvalue analysis.

\section{Appendix: Machine Model}

$$
\begin{aligned}
& \dot{\delta}=\omega_{b}\left(\omega_{i^{-}} 1\right) \\
& \dot{\boldsymbol{\omega}}_{i}=\left(P_{m}-P_{e i}-D_{i}\left(\omega_{i}-1\right)\right) / M_{i} \\
& \dot{\boldsymbol{E}}_{q i}^{\prime}=\left(\boldsymbol{E}_{f d i}-\left(x_{d i}-x_{d i}^{\prime}\right) i_{d i}-E_{q i}^{\prime}\right) / T_{d o i}^{\prime} \\
& \dot{\boldsymbol{E}}_{d i}^{\prime}=\left(\left(x_{q i}-x_{d i}{ }_{d i}\right) i_{q i}-\boldsymbol{E}_{d i}{ }_{d i}\right) / \boldsymbol{T}_{q o i} \\
& T_{e i}=E_{q i}^{\prime} i_{q i}-\left(x_{q i}-x_{d i}\right) i_{d i} i_{q i} \\
& \delta \quad \text { rotor angle } \\
& \omega \quad \text { rotor speed } \\
& P_{m} \quad \text { mechanical input power } \\
& P_{e} \quad \text { electrical output power } \\
& \boldsymbol{E}_{q} \quad \text { internal voltage behind } \boldsymbol{x}_{d i} \\
& E_{f d} \quad \text { equivalent excitation voltage } \\
& T_{e} \quad \text { electric torque } \\
& T_{d o} \quad \text { time constant of excitation circuit } \\
& T_{q o} \quad \text { regulator time constant } \\
& v_{r e f} \text { reference voltage } \\
& v \quad \text { terminal voltage }
\end{aligned}
$$

\section{REFERRENCES}

[1] Padiyar K.R., Power System Dynamics Stability and Control', Anshan Publishers, $2^{\text {nd }}$ edition.

[2] Anderson P. M., Fouad, A.A., Power System Control and Stability, IEEE series on power engineering, $2^{\text {nd }}$ edition.

[3] Larsen E. and Swann D., Applying power system stabilizers, IEEE Trans. Power App. Systems, Vol. PAS100, 1981, pp.3017-3046.

[4] Cao Y., Jiang L., Cheng S., Chen D., Malik O.P. and Hope G.S., " A nonlinear variable structure stabilizer for power system stability", IEEE Trans. Energy Conversion, vol. 9, Sept. 1994,pp. 489-495.

[5] Hiyama T. and Sameshima T., "Fuzzy logic control scheme for on-line stabilization of multimachine power system,” Fuzzy Sets Sys., vol. 39, 1991 pp. 181-194.

[6] Abido M.A. and Abdel-Magid Y.L., "A hybrid neurofuzzy power system stabilizer for multi-machine power systems," IEEE Trans. Power Syst., vol. 13, Nov. 1998pp. 1323-1330.

[7] D. Xia and G. T. Heydt, "Self-tuning controller for generator excitation control," IEEE Trans. Power App. Syst., 1983, pp. 1877-1885.

[8] P. Kundur, M. Klein, G. J. Rogers, and M. S. Zywno, Application of power system stabilizers for enhancement of overall system stability, IEEE Trans. on Power Systems. Vol. PAS-108, 1989, pp.614-626.

[9] R. J. Fleming, M. A. Mohan, K Parvatism, “ Selection of parameters of stabilizers in multi-machine power systems", IEEE Trans, PAS, vol. 100, no. 5,1981, pp. 2329-2333.

[10] Mahdiyeh Eslami, Hussain Shareef and Azah Mohamed, "Optimal tuning of power system stabilizers using modified particle swarm optimization", Proceedings of the 14th International Middle East Power Systems 
Conference (MEPCON'10), Cairo University, Egypt, December 19-21, 2010.

[11] M. A. Abido, "A novel approach to conventional power system stabilizer design using tabu search," Int. J. Electr. Power Energy Syst., vol. 21, 1999, pp. 443-454.

[12] Y. L. Abdel-Magid and M. A. Abido, "Optimal multiobjective design of robust power system stabilizers using genetic algorithms," IEEE Transactions on Power Systems, vol. 18, 2003,pp. 1125-1132.

[13] A. L. B. Do Bomfim, et al., "Simultaneous tuning of power system damping controllers usinggenetic algorithms," IEEE Trans. Power Syst.,vol. 15,2000, pp. 163-169.

[14] M. A. Abido, Robust design of multi-machine power system stabilizers using simulated annealing, IEEE Trans. on Energy Conversion, Vol.15, No. 3, 2003, pp. 297-304.

[15] M. A. Abido, Y. L. Abdel-Magid, Optimal design of power system stabilizers using evolutionary programming, IEEE Trans. on Energy Conversion, Vol.17, No. 4,2002, pp.429-436.

[16] P. Zhang and A. H. Coonick, "Coordinated synthesis of PSS parameters in multi-machine powersystems using the method of inequalities applied to genetic algorithms," IEEE Trans. Power Syst., vol. 15, 2000, pp. 811-816.

[17] A.R. Mehrabian, C. Lucas, A novel Numerical Optimization Algorithm Inspired from Weed Colonization, Ecological Informatics, 2006, vol 1.pp-355366.

[18] A.R. Mehrabian, A. Yousefi-Koma, Optimal Positioning of Piezoelectric Actuators on a Smart Fin using Bioinspired Algorithms, Aerospace Science and Technology,2007, vol 11, pp 174-182.

[19] A. R. Mallahzadeh ,S. Es'haghi, A Alipour, “ Design of an E shaped MIMO Antenna using IWO Algorithm for Wireless Application at 5.8 Ghz", Progress in Electromagnetic Research,PIER 90, 2009,187-203.
[20] X. Zhang, Y. Wang, G. Cui, Y. Niu, J. Xu, Application of a novel IWO to the design of encoding sequence for DNA computing, Comput. Math. Appl. 57, Jun 2009, pp. 2001-2008,

[21] H. Sepehri Rad , C. Lucas, “ A Recommender System based on Invasive Weed Optimization Algorithm", IEEE Congress on Evolutionary Computation, CEC 2007,pp 4297-4304.

[22] M. Shaheri-Ardakani, M. Rshanaei, A. Rahimi-Kian, C. Lucas, "A study of electricity market dynamics using Invasive Weed Colonization Optimization," in Proc. IEEE Symp. Comput.Intell. Games, 2008, pp.276-282.

[23] P. W. Sauer and M. A. Pai, Power System Dynamics and Stability.(Englewood Cliffs, NJ: Prentice-Hall, 1998.)

[24] J. Kennedy and R.C. Eberhart, "Particle swarm optimization," in Proc.IEEE Int. Conference on Neural Networks, Piscataway, NJ, pp. 1942-1948, 1995.

[25] Y. Shi and R. C. Eberhart, "A modified particle swarm optimizer," in Proc. IEEE Int. Conference on Evolutionary Computation, Piscataway, NJ, pp 69-73, 1998.

[26] M. Meissner, M. Schmuker, and G. Schneider, "Optimized Particle Swarm Optimization (OPSO) and its application to artificial neural network training," $B M C$ Bioinformatics, vol. 7, no. 125, 2006.

[27] Z. Cui1, J. Zeng, and G. Sun, "A Fast Particle Swarm Optimization," Int. J. of Innovative Computing, Information and Control, vol. 4, no. 6, pp. 1365-1380, 2006.

[28] S. Karimkashi, Ahmed A. Kishk, "Invasive Weed Optimization and its Features in Electromagnetics", IEEE Transactions on Antenna and Propagation, Vol. 58, No. 4, April 2010, pp. 1269-1278.

[29] Kundur P., Power System Stability And Control (McGraw-Hill Press, 1994). 